\title{
Clinical Study \\ Effect of Traditional Chinese Medicine on Inflammatory Mediators in Pediatric Asthma
}

\author{
Hui Du, Yonghong Wang, Yumin Shi, Jian Yu, Wen Sun, and Yiqun Zhang \\ Department of Traditional Chinese Medicine, Children's Hospital of Fudan University, No. 399, Wanyuan Road, \\ Shanghai 201102, China
}

Correspondence should be addressed to Yonghong Wang; wyhekyy@126.com

Received 17 March 2016; Accepted 19 May 2016

Academic Editor: Kyung H. Kim

Copyright (C) 2016 Hui Du et al. This is an open access article distributed under the Creative Commons Attribution License, which permits unrestricted use, distribution, and reproduction in any medium, provided the original work is properly cited.

Objective. To observe the effects of empirical prescriptions of traditional Chinese medicine (TCM) on inflammatory mediators in pediatric asthma and to explore the underlying molecular mechanism in the treatment of asthma. Methods. A total of 182 children with asthma were randomly placed into either the TCM group $(n=97)$ or the salbutamol and montelukast (SM) group $(n=85)$. Patients in the TCM group were treated with a series of empirical prescriptions of TCM, while those in the SM group received salbutamol and montelukast. Both groups received their respective treatment for 12 weeks. There were 35 patients in TCM group and 34 patients in SM group providing venous blood. Real-time PCR was used to determine the mRNA expression levels of interleukin- (IL-) 10, IL-17, matrix metalloproteinase-9 (MMP-9), and transforming growth factor $\beta 1$ (TGF- $\beta 1$ ) in peripheral blood mononuclear cells before and after treatment. Enzyme-linked immunosorbent assay was used to measure the levels of IL-10, IL-17, MMP-9, and TGF- $\beta 1$ in peripheral blood before and after treatment. Results. The mRNA expression of TGF- $\beta 1$ in the SM group was downregulated $(P=0.00)$ after treatment. No significant differences were found between the TCM group and the SM group after treatment $(P>0.05)$. In the TCM group, the levels of IL-10, IL-17, and MMP-9 significantly decreased after treatment $(P=0.01$, 0.04 , and 0.03 , resp.). In the SM group, IL-17, MMP-9, and TGF- $\beta 1$ levels significantly decreased after treatment $(P=0.00,0.03$, and 0.00 , resp.). There was no significant difference between the two groups regarding the levels of IL- 10 , IL-17, TGF- $\beta 1$, and MMP-9 $(P>0.05)$. The difference of the level of IL-17 was negatively correlated with the change of C-ACT score in TCM group and SM group. Conclusion. TCM has a regulatory effect on the balance of some inflammatory mediators in pediatric asthma.

\section{Introduction}

Asthma is a heterogeneous disease that is usually characterized by chronic airway inflammation. It involves participation of eosinophil granulocytes, T cells, mast cells, and cytokines. Asthma is the most common chronic childhood disease and the leading cause of childhood morbidity from chronic disease [1]. Although the pathogenesis of asthma is not yet clear, it is believed that an imbalanced ratio of Th1 and Th2 cell function is an important mechanism of this disease [2]. In addition, type 17 helper (Th17) cells play an important role in the development of asthma [3]. Studies showed that matrix metalloproteinase-9 (MMP-9) and transforming growth factor-beta 1 (TGF- $\beta 1$ ) are also closely related to airway inflammation and airway remodeling $[4,5]$.

Traditional Chinese medicine (TCM) treatments for asthma have achieved good results. A previous study showed that empirical prescriptions of TCM effectively control asthma exacerbation and improve lung function [6]. However, the mechanism of the empirical prescriptions is unclear. This study was designed to explore the regulatory effect of empirical prescriptions of TCM on the inflammatory mediators and cytokines related to asthma in children in order to provide a theoretical basis for clinical practice.

\section{Materials and Methods}

2.1. Study Design. This study was a single-blind randomized controlled trial conducted at the Children's Hospital of Fudan University. Conventional treatment (salbutamol and montelukast) for asthma constituted the positive control treatment for the determination of the clinical effect of TCM treatment regimen on pediatric asthma. This study obtained approval from the Ethics Committee of Children's Hospital 
of Fudan University (number [2013] 065) and registered at http://clinicaltrials.gov/ct2/show/NCT02341573.

2.2. Inclusion and Exclusion Criteria. This study followed diagnostic criteria established in 2008, in accordance with the "standards in the Guideline of Childhood Bronchial Asthma" by the Pulmonology Group of the Pediatric Branch of the Chinese Medical Association [7]. TCM syndrome was defined according to "standards for the diagnosis and clinical effects on TCM syndrome" by the State Administration of Traditional Medicine [8]. Patients aged 3-12 years old with a syndrome meeting the above criteria for the diagnosis of pediatric asthma and cough-variant asthma were enrolled into the study. Asthmatic episodes were catalogued according to TCM syndrome differentiation as follows: a form of phlegm-heat obstructing the lungs or cold fluid retention in the lungs, lung, and spleen deficiencies or deficiency of kidneys in clinical remission stage of asthma. Patients with congenital diseases of the respiratory system, tumors, immunodeficiency diseases, or cardiovascular diseases were excluded.

2.3. Subjects. A total of 182 children with asthma were recruited between March 2013 and December 2014. They were randomly placed (using the random number table method) into either the SM (salbutamol and montelukast) group ( $n=$ $85)$ or the TCM group $(n=97)$. All the children who signed the consent and were willing to provide venous blood were enrolled and then randomly assigned to separate groups. At the end of this trial, there were 35 patients in TCM group and 34 patients in SM group providing venous blood before and after treatment. There were 17 girls and 18 boys with a mean age of $5.0 \pm 1.6$ years and a mean disease course of $8.3 \pm 6.4$ months in the TCM group. In SM group, there were 15 girls and 20 boys with a mean age of $5.0 \pm 1.2$ years and a mean disease course of 7.6 \pm 6.5 months; one girl was lost to followup. There were no significant differences between two groups regarding age, gender, and disease course.

\subsection{Interventions}

TCM Group. Patients received a series of empirical TCMoriented treatments. The initial treatment was Shegan Mixture (hospital preparations, Shanghai Liantang Pharmaceutical Co., batch number 20130101) corresponding to the patients in acute exacerbation stage of asthma. The composition of this prescription was as follows: $9 \mathrm{~g}$ ephedrine, $9 \mathrm{~g}$ almond, $6 \mathrm{~g}$ Belamcanda chinensis, $9 \mathrm{~g}$ Rorippa indica, $9 \mathrm{~g}$ Radix scutellariae, and $9 \mathrm{~g}$ batryticated silkworm. It was administered three times daily in a $10 \mathrm{~mL}$ mixture, over a $7 \mathrm{~d}$ treatment course. At follow-up visits during the fourth and eighth weeks, the prescription was adjusted according to TCM syndrome and SC scores. Patients in chronic persistent stage of asthma were treated with Shegan Mixture and Astragalus kidney tonic mixture. Patients in clinical remission were treated with Astragalus kidney tonic mixture comprised of $9 \mathrm{~g}$ Astragalus, $9 \mathrm{~g}$ atractylodes, $15 \mathrm{~g}$ Chinese yam, $9 \mathrm{~g}$ Radix pseudostellariae, $12 \mathrm{~g}$ Poria cocos, and $9 \mathrm{~g}$ medicinal Indian mulberry. Complementary herbs were added or removed according to presenting symptoms. For example, magnolia flower and Fructus xanthii were administered for nasal congestion and sneezing, ephedrine root and floating wheat were administered for hyperhidrosis, and Semen raphani and F. forsythia were prescribed for dry feces. These treatments were administered for 12 weeks.

The criteria for the quality of the herbs used were in accordance with the Chinese pharmacopeia (2005) [9].

SM Group. Patients with acute exacerbation of asthma were mainly treated with bronchial relaxants, salbutamol sulfate sustained release capsules (Ethypharm Pharmaceutical Co., Ltd., Shanghai, China, batch number 121201), 2-4 mg twice daily for $7 \mathrm{~d}$. Patients in clinical remission were treated with leukotriene receptor antagonists, montelukast sodium chewable tablets (MSD pharmaceutical Co., Ltd., batch number 120054), 4-5 mg nightly for 3 months. Patients with chronic persistence of asthma were treated with salbutamol and montelukast.

Antibiotics or atomization treatment was administered in cases of acute bacterial infection and severe wheezing.

2.5. Outcomes. The outcomes were changes in the levels of IL-10, IL-17, MMP-9, and TGF- $\beta 1$ in peripheral blood, and the mRNA expression levels of those cytokines in peripheral blood mononuclear cells (PBMCs) before and after 12 weeks of treatment, as well as changes in children asthma control test (C-ACT) scores before and after 12 weeks of treatment.

2.5.1. C-ACT. C-ACT is a widely used asthma control assessment tool that is recognized by the Global Initiative for Asthma (GINA). This test includes seven questions and is divided into two parts: the first four questions are selfadministered by the child; the last three questions are completed by a caregiver on the basis of the child's condition during the previous 4 weeks. With a total of 27 points, scores of 23 to 27 are classified as well controlled; 20 22 as partly controlled; and 0 19 as very poorly controlled asthma. The quality of this test was highly controlled by specialist.

2.5.2. Specimen Collection and Processing. Peripheral blood samples $(2 \mathrm{~mL})$ were taken aseptically from asthmatic children and injected into the EDTA anticoagulation tubes with gentle shaking. Plasma was separated by centrifugation for $5 \mathrm{~min}$ at a speed of $2500 \mathrm{rpm}$ and frozen for later analysis. PBMCs were separated with the Ficoll method (Huajing Biological Engineering Co. Ltd., Shenzhen, China).

2.5.3. Real-Time Polymerase Chain Reaction (Real-Time PCR). Total RNA was extracted from PBMCs using Trizol RNA extraction reagent (Life Technologies, Carlsbad CA, USA) and reverse-transcribed into cDNA (PCR MasterMix kit, Toyobo Co., Ltd., Osaka, Japan). Primers of each gene were designed using Primer Premier 5.0 (PREMIER Biosoft, Palo Alto, CA, USA) according to the cDNA sequences obtained from the National Center for Biotechnology Information. The primer sequences and amplicon sizes are shown in Table 1. Quantitative real-time PCR (qRT-PCR) was performed using SYBR Green Premix Ex Taq in a Mastercycler ep realplex instrument (Eppendorf AG., Hamburg, Germany). The PCR 
TABLE 1: Primer sequences and amplicon sizes.

\begin{tabular}{llc}
\hline Gene name & Primer sequence $\left(5^{\prime}\right.$ to $\left.3^{\prime}\right)$ & $\begin{array}{c}\text { Amplicon } \\
\text { size }(\mathrm{bp})\end{array}$ \\
\hline GAPDH-F & CTCTCTGCTCCTCCTGTTCGAC & 69 \\
GAPDH-R & TGAGCGATGTGGCTCGGCT & \\
IL10-F & GATGCCTTCAGCAGAGTGAA & 105 \\
IL10-F & GCAACCCAGGTAACCCTTAAA & \\
IL17A-F & GCTGATGGGAACGTGGACTA & 125 \\
IL17A-R & CCCACGGACACCAGTATCTT & \\
TGF $\beta-F$ & TGGTGGAAACCCACAACGAA & 113 \\
TGF $\beta-R$ & GAGCAACACGGGTTCAGGTA & \\
MMP9-F & CATCGTCCACCGGACTCAAA & 179 \\
MMP9-R & AAACCGAGTTGGAACCACGA & \\
\hline
\end{tabular}

protocol was as follows: predenaturation for $20 \mathrm{~min}$ at $95^{\circ} \mathrm{C}$ followed by denaturation for $30 \mathrm{~s}$ at $95^{\circ} \mathrm{C}$, annealing for $30 \mathrm{~s}$ at $54-55^{\circ} \mathrm{C}$, and extension for $40 \mathrm{~s}$ at $72^{\circ} \mathrm{C}$ for $40 \mathrm{cycles}$. The relative amount of target gene mRNA was normalized to GAPDH.

2.5.4. Enzyme-Linked Immunosorbent Assey (ELISA). Plasma was obtained by sample centrifugation and anticoagulation. The content of IL-10, IL-17, MMP-9, and TGF- $\beta 1$ was detected with a double antibody sandwich enzyme-linked immunosorbent assay (ELISA) kit, according to the kit instructions. The kits were supplied by RayBiotech, Inc., American. Optical density (OD) values were detected with an enzyme-labeled meter (Perlong Medical Instrument Co., Ltd., Beijing, China) at a wavelength of $450 \mathrm{~nm}$. The diluted concentration standards used for IL-10, IL-17, MMP-9, and TGF- $\beta 1$ were $150,75,37.5,18.75,9.38,4.69,2.34$, and $0 \mathrm{pg} / \mathrm{mL}$, $6000,3000,1500,750,375,187.5,93.75$, and $0 \mathrm{pg} / \mathrm{mL}, 6000$, 2000, 666.7, 222.2, 74.07, 24.6, 8.23, and $0 \mathrm{pg} / \mathrm{mL}$, and 60, 20, $6.667,2.222,0.741,0.247,0.0823$, and $0 \mathrm{ng} / \mathrm{mL}$, respectively.

2.6. Statistical Analysis. Intention-to-treat analysis was performed. Demographic data were analyzed to identify baseline equivalencies and differences between the two groups. Continuous variables were presented as mean \pm standard deviation (SD), evaluated by using Student's $t$-tests based on normal distribution. Data presented as abnormal distribution were evaluated by using $t$-tests after log transformation. Correlation analysis of normal distribution variables was evaluated by using Spearman. All data were analyzed using SPSS version 19 software. $P<0.05$ was considered statistically significant.

\section{Results}

3.1. Comparison of the mRNA Expression Levels of $I L-10, I L-$ $17, T G F-\beta 1$, and MMP-9. No significant difference was found between the TCM group and the SM group after treatment $(P>0.05)$ (Table 2$)$. In the SM group, the mRNA expression of TGF- $\beta 1$ was downregulated $(P=0.00)$ after treatment. There was no significant difference of the mRNA expression of IL-10, IL-17, TGF- $\beta 1$, and MMP-9 after treatment in TCM group (Table 3 ).

3.2. Comparison of the Levels of $I L-10, I L-17, T G F-\beta 1$, and MMP-9 in Peripheral Blood. There was no significant difference in the levels of IL-10, IL-17, TGF- $\beta 1$, and MMP-9 in peripheral blood between the TCM group and the SM group after treatment $(P>0.05)$. In the TCM group, the levels of IL-10, IL-17, and MMP-9 were significantly decreased after treatment $(P=0.01,0.04$, and 0.03 , resp.). In the SM group, IL-17, MMP-9, and TGF- $\beta 1$ levels were significantly decreased after treatment $(P=0.00,0.03$, and 0.00 , resp.) (Tables 4 and 5).

3.3. Correlation between C-ACT Score and the Levels of IL$10, I L-17, T G F-\beta 1$, and MMP-9. The TCM group showed significantly higher C-ACT scores after 12 weeks of treatment than those of patients before treatment (after $22.5 \pm 1.9$ versus before $16.5 \pm 1.9, P<0.001)$. In the SM group, C-ACT scores significantly increased after 12 weeks of treatment (after $22.1 \pm$ 2.0 versus before $17.0 \pm 2.2, P<0.001$ ).

The difference of the level of IL-17 was negatively correlated with the change of C-ACT score in TCM group and SM group. No correlation was found between the difference of C-ACT score and the levels of IL-10, TGF- $\beta 1$, and MMP-9 in both groups (Tables 6 and 7).

\section{Discussion}

Asthma is a chronic inflammatory disease, in which a variety of cells and cytokines play major roles. Previous studies have indicated that the pathogenesis of airway inflammation in asthma patients may be related to the imbalance of Th1/Th2. Increased number and activation status of Th2 cells cause elevated secretion of Th2-type cytokines (IL-4, IL-5, and IL-10), which are critical factors in the initiation and maintenance of airway inflammation $[10,11]$. In previous study, we had found that series empirical prescriptions have a regulatory effect on leukotriene receptor gene expression and the imbalance of Th1/Th2 immune cells in the process of asthmatic attacks [12] and also observed that empirical prescriptions of TCM for the treatment could effectively control asthma attacks and improve multiple lung function indices [6]. To elucidate the immunologic mechanism of TCM in the treatment of asthma, we examined the expression of some more cytokines related to asthma, including IL-10, IL-17, TGF- $\beta 1$, and MMP-9 in this study.

Previously studies showed that the prescription for TCM group, Shegan Mixture, was derived from the Belamcandae and Ephedrine Decoction. Previous study showed that Belamcandae and Ephedrine Decoction can improve the immune function of the patients (effectively preventing hypersensitivity) [13], improve clinical effects in the treatment of pediatric cough-variant asthma, and modify IL10 and IL-13 serum levels [14]. Luo et al. [15] found that compared with an asthma model group, the Belamcandae and Ephedrine Decoction group showed a reduced expression of TGF- $\beta 1$, decreased thickness of bronchial wall and smooth muscle, and a reduction in inflammatory cells infiltration. In 
TABLE 2: Comparison of the mRNA expression levels of IL-10, IL-17, TGF- $\beta 1$, and MMP-9 between the TCM and SM groups before and after treatment (mean $\pm \mathrm{SD})$.

\begin{tabular}{lccccc}
\hline \multirow{2}{*}{ Variables } & \multicolumn{3}{c}{ Before treatment } & \multicolumn{2}{c}{ After treatment } \\
& TCM group $(n=35)$ & SM group $(n=34)$ & $P$ value & TCM group $(n=35)$ & SM group $(n=34)$ \\
\hline IL-17 & $5.3 \pm 8.2$ & $4.2 \pm 6.8$ & 0.60 & $8.0 \pm 7.6$ & $8.8 \pm 11.2$ \\
TGF- $\beta 1$ & $196.6 \pm 94.9$ & $227.7 \pm 130.5$ & 0.32 & $170.2 \pm 83.0$ & $120.3 \pm 60.1$ \\
MMP-9 & $2.5 \pm 3.5$ & $1.7 \pm 3.6$ & 0.49 & $2.6 \pm 4.5$ & 0.86 \\
IL-10 & $0.5 \pm 0.3$ & $0.5 \pm 0.5$ & 0.33 & $0.6 \pm 0.6$ & 0.09 \\
\hline
\end{tabular}

TABLE 3: Comparison of the mRNA expression levels of IL-10, IL-17, TGF- $\beta 1$, and MMP-9 in the TCM and SM groups before and after treatment $($ mean $\pm \mathrm{SD})$.

\begin{tabular}{lccccc}
\hline \multirow{2}{*}{ Variables } & \multicolumn{2}{c}{ TCM group $(n=35)$} & \multicolumn{3}{c}{ SM group $(n=34)$} \\
& Before & After & $P$ value & Before & After \\
\hline IL-17 & $5.3 \pm 8.2$ & $8.0 \pm 7.6$ & 0.39 & $4.2 \pm 6.8$ & $8.8 \pm 11.2$ \\
TGF- $\beta 1$ & $196.6 \pm 94.9$ & $170.2 \pm 83.0$ & 0.44 & $227.7 \pm 130.5$ & $120.3 \pm 60.1$ \\
MMP-9 & $2.5 \pm 3.5$ & $2.6 \pm 4.5$ & 0.97 & $1.7 \pm 3.6$ & $1.8 \pm 3.3$ \\
IL-10 & $0.5 \pm 0.3$ & $0.6 \pm 0.6$ & 0.63 & $0.5 \pm 0.5$ & 0.00 \\
\hline
\end{tabular}

TABLE 4: Comparison of the peripheral blood levels of IL-10, IL-17, TGF- $\beta 1$, and MMP-9 between the TCM group and SM group before and after treatment $(\mathrm{pg} / \mathrm{mL}, \mathrm{MMP}-9 \mathrm{ng} / \mathrm{mL}$, mean $\pm \mathrm{SD})$.

\begin{tabular}{|c|c|c|c|c|c|c|}
\hline \multirow{2}{*}{ Variables } & \multicolumn{3}{|c|}{ Before treatment } & \multicolumn{3}{|c|}{ After treatment } \\
\hline & TCM group $(n=35)$ & SM group $(n=34)$ & $P$ value & TCM group $(n=35)$ & SM group $(n=34)$ & $P$ value \\
\hline IL-17 & $1919.7 \pm 1868.4$ & $1845.7 \pm 1552.3$ & 0.87 & $1072.3 \pm 689.8$ & $928.1 \pm 440.2$ & 0.54 \\
\hline TGF- $\beta 1$ & $1003.3 \pm 914.1$ & $1512.3 \pm 997.7$ & 0.02 & $1079.2 \pm 728.1$ & $634.6 \pm 391.3$ & 0.07 \\
\hline MMP-9 & $387.3 \pm 119.3$ & $423.1 \pm 170.4$ & 0.42 & $301.4 \pm 75.2$ & $331.1 \pm 99.3$ & 0.42 \\
\hline IL-10 & $43.2 \pm 61.9$ & $22.6 \pm 12.9$ & 0.08 & $12.4 \pm 5.1$ & $16.0 \pm 13.2$ & 0.36 \\
\hline
\end{tabular}

TABLE 5: Comparison of the peripheral blood levels of IL-10, IL-17, TGF- $\beta 1$, and MMP-9 in the TCM and SM groups before and after treatment (pg/mL, MMP-9 ng/mL, mean $\pm \mathrm{SD})$.

\begin{tabular}{lccccc}
\hline \multirow{2}{*}{ Variables } & Before & TCM group $(n=35)$ & & \multicolumn{2}{c}{ SM group $(n=34)$} \\
& After & $P$ value & Before & After \\
\hline IL-17 & $1919.7 \pm 1868.4$ & $1072.3 \pm 689.8$ & 0.04 & $1845.7 \pm 1552.3$ & $928.1 \pm 440.2$ \\
TGF- $\beta 1$ & $1003.3 \pm 914.1$ & $1079.2 \pm 728.1$ & 0.81 & $1512.3 \pm 997.7$ & $634.6 \pm 391.3$ \\
MMP-9 & $387.3 \pm 119.3$ & $301.4 \pm 75.2$ & 0.03 & $423.1 \pm 170.4$ & $331.1 \pm 99.3$ \\
IL-10 & $43.2 \pm 61.9$ & $12.4 \pm 5.1$ & 0.01 & $22.6 \pm 12.9$ & 0.00 \\
\hline
\end{tabular}

TABLE 6: Correlation analysis between changes of C-ACT scores and the levels of IL-10, IL-17, TGF- $\beta 1$, and MMP-9 in TCM group.

\begin{tabular}{lccccc}
\hline Variables & d_IL10 & d_IL17 & d_MMP-9 & d_ACT & d_TGF $\beta 1$ \\
\hline d_IL10 & 1.0000 & & & & \\
d_IL17 & $-0.9000^{*}$ & 1.0000 & & & \\
d_MMP9 & -0.2000 & -0.1000 & 1.0000 & & \\
d_C-ACT & 0.8721 & $-0.9747^{*}$ & 0.2052 & 1.0000 & \\
d_TGF $\beta 1$ & -0.6000 & 0.5000 & -0.4000 & -0.5643 & 1.0000 \\
\hline${ }^{*} P<0.05$. & & & & &
\end{tabular}

our study, the level of IL-10 decreased after 12 weeks of TCM treatment, suggesting that TCM may inhibit airway inflammation by reducing the secretions of IL-10 to control asthma attacks. We were not able to detect differences following 12
TABLE 7: Correlation analysis between changes of C-ACT scores and the levels of IL-10, IL-17, TGF- $\beta 1$, and MMP-9 in SM group.

\begin{tabular}{lccccc}
\hline Variables & d_IL10 & d_IL17 & d_MMP9 & d_C-ACT & d_TGF $\beta 1$ \\
\hline d_IL10 & 1.0000 & & & & \\
d_IL17 & 0.1190 & 1.0000 & & & \\
d_MMP9 & 0.4762 & 0.0238 & 1.0000 & & \\
d_C-ACT & -0.4524 & $-0.7143^{*}$ & -0.2619 & 1.0000 & \\
d_TGF $\beta 1$ & 0.4048 & 0.0000 & -0.2381 & -0.1429 & 1.0000 \\
\hline${ }^{*} P<0.05$. & & & & &
\end{tabular}

weeks of TCM treatment for TGF- $\beta 1$ serum concentration. The different outcomes of the various studies could be attributed to the different techniques used to quantify the secreted levels of inflammatory mediators. 
Recent studies have shown that the imbalance of Th17/Treg is also related to asthma. IL-17 plays an important role in the processes of airway remodeling and airway inflammation by chemotaxis and activation of neutrophils [16]. MMP-9 also participates in airway remodeling by degrading collagen and promoting epithelial cell migration [17]. Miao et al. [18] found that pingchuan mixture can reduce the expression of IL-17 and MMP-9 in lung tissue to improve the process of airway remodeling in asthma. In our study, empirical prescriptions of TCM reduced the levels of IL-17 and MMP-9 in peripheral blood, suggesting that it also has a regulatory effect on the secretion of IL-17 and MMP-9.

At the same time, we also recorded the C-ACT score to assess asthma control. The results showed that the C-ACT scores increased after 12 weeks of TCM treatment, similar to findings of our previous study. Liu et al. [19] found that CACT score can reflect the clinical symptoms well, and this conclusion has also been proved in our study. The results also showed that the difference of the level of IL-17 was negatively correlated with the change of C-ACT score in TCM group. We speculate that empirical prescriptions of TCM might inhibit airway inflammation by decreasing the secretion of IL17.

Interestingly, no differences in the levels of IL-10, IL17, and MMP-9 mRNA were found after treatment in TCM group, with the reduction of those serum levels. These results were somewhat inconsistent with our expectations, so we tried to analyze possible causes of the results. Empirical prescriptions of TCM may affect the levels of cytokine (IL-10, IL-17, and MMP-9) in the process of translation. Besides, the expression process of cytokines is complex, and the number of samples in our study is limited, which may result in the deviation of the results.

The present study demonstrates that empirical prescriptions of TCM can effectively control asthma attacks and reduce the levels of IL-10, IL-17, and MMP-9 in peripheral blood.

\section{Conclusion}

In conclusion, these results demonstrate that empirical prescriptions of TCM have a regulatory effect on the secretion of some inflammatory mediators in pediatric asthma and can help understand the immunologic mechanism of the treatment of asthma.

\section{Competing Interests}

The authors declare that there is no conflict of interests regarding the publication of this paper.

\section{Authors' Contributions}

All authors contributed equally to this work.

\section{Acknowledgments}

The authors thank all patients for their participation. Special thanks are also extended to colleagues at the Children's
Hospital of Fudan University. This study is supported by grants from the Major Research Project of Shanghai Traditional Chinese Medicine Three-Year Action Plan (no. ZYSNXD-CC-ZDYJ034), Development Project of Shanghai Peak Disciplines-Integrated Chinese and Western Medicine, and the Shanghai Science and Technology Research Program (no. 12401905500).

\section{References}

[1] M. Masoli, D. Fabian, S. Holt, and R. Beasley, "The global burden of asthma: executive summary of the GINA Dissemination Committee report," Allergy, vol. 59, no. 5, pp. 469-478, 2004.

[2] H. Z. Chen and C. M. Zhu, "The immunological pathogenesis of bronchial asthma," Journal of Applied Clinical Pediatrics, no. 12, pp. 1015-1017, 2004.

[3] H. J. Li, Q. L. Gai, L. Dong et al., "Preliminary study on Th17 lymphocytes in the airway inflammation of asthmatic mice," Chinese Journal of Cellular and Molecular Immunology, vol. 27, no. 1, pp. 53-55, 2011.

[4] G. Chen and N. Khalil, "TGF- $\beta 1$ increases proliferation of airway smooth muscle cells by phosphorylation of map kinases," Respiratory Research, vol. 7, article 2, 2006.

[5] J. J. Atkinson and R. M. Senior, "Matrix metalloproteinase-9 in lung remodeling," American Journal of Respiratory Cell and Molecular Biology, vol. 28, no. 1, pp. 12-24, 2003.

[6] Y. H. Wang, Y. M. Shi, J. Yu et al., "Clinical observation on stage treatment with experienced Chinese herbal formulas for 45 cases of pediatric asthma," Journal of Traditional Chinese Medicine, vol. 53, no. 24, pp. 2105-2108, 2012.

[7] Group of Pneumatology. Branch Association of Pediatrics. Chinese Medical Association, "Guideline of childhood bronchial asthma, 2008," Chinese Journal of Pediatrics, vol. 46, no. 10, pp. 745-753, 2008 .

[8] State Administration of Traditional Chinese Medicine, "Standards for diagnosis and clinical effect of Chinese medical symptom," Journal of Traditional Chinese Medicine Management, vol. 6, no. 2, pp. 286-287, 1994.

[9] Pharmacopoeia Committee of People's Republic of China, Chinese Pharmacopoeia, Chemical Industry Press, Beijing, China, 2005.

[10] A. S. McKee and E. J. Pearce, "CD $25^{+} \mathrm{CD} 4^{+}$cells contribute to Th2 polarization during helminth infection by suppressing Th1 response development," Journal of Immunology, vol. 173, no. 2, pp. 1224-1231, 2004.

[11] S. Finotto and L. Glimcher, "T cell directives for transcriptional regulation in asthma," Springer Seminars in Immunopathology, vol. 25, no. 3-4, pp. 281-294, 2004.

[12] S. H. Li, Y. H. Wang, Y. M. Shi et al., "Regulatory effects of stage-treatment with established Chinese herbal formulas on inflammatory mediators in pediatric asthma," Journal of Traditional Chinese Medicine, vol. 33, no. 6, pp. 727-732, 2013.

[13] S. J. Tan, H. Ai, and W. Y. Wang, "Experiment research of revised belamcandae and ephedrine decoction on allergic asthma," Journal of Traditional Chinese Medicine, vol. 41, no. 5, pp. 282283, 2000.

[14] Z. X. Chen and G. H. Hu, "Effect of modified Shegan Mahuang decoction on cytokines in children patients with cough and variant asthma," Chinese Journal of Integrated Traditional and Western Medicine, vol. 30, no. 2, pp. 208-209, 2010. 
[15] Y. C. Luo, J. L. Han, and T. Zhang, "Influence of Belamcandae and Ephedra Decoction on cytokines expression in lung tissue of bronchial asthma rat," Chinese Journal of Gerontology, vol. 9, pp. 1056-1057, 2009.

[16] O. Prause, S. Bozinovski, G. P. Anderson, and A. Lindén, "Increased matrix metalloproteinase- 9 concentration and activity after stimulation with interleukin-17 in mouse airways," Thorax, vol. 59, no. 4, pp. 313-317, 2004.

[17] N. A. Hasaneen, R. Z. Lin, R. A. Panettieri, S. Zucker, G. G. Vaday, and H. D. Foda, "Angiogenesis is induced by airway smooth muscle strain," American Journal of Physiology - Lung Cellular and Molecular Physiology, vol. 293, no. 4, pp. L1059L1068, 2007.

[18] L. Y. Miao, Z. L. Han, Z. H. Qu, and L. Xu, "The effect of pingchuan mixture on interleukin-17 in bronchoalveolar lavage fluid and airway remodeling of asthma mice," Chinese Journal of Applied Clinical Pediatrics, vol. 28, no. 16, pp. 1250-1252, 2013.

[19] A. H. Liu, R. Zeiger, C. Sorkness et al., "Development and crosssectional validation of the Childhood Asthma Control Test," Journal of Allergy and Clinical Immunology, vol. 119, no. 4, pp. 817-825, 2007. 


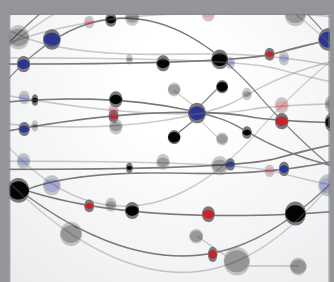

The Scientific World Journal
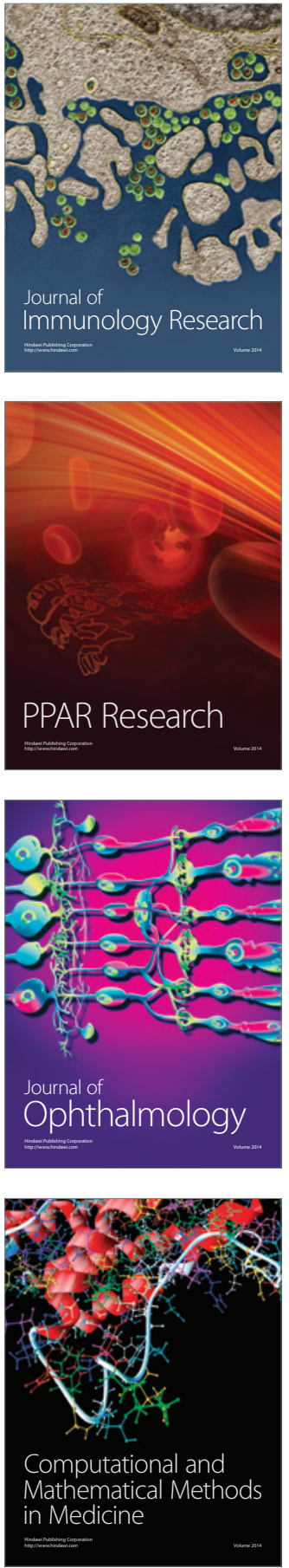

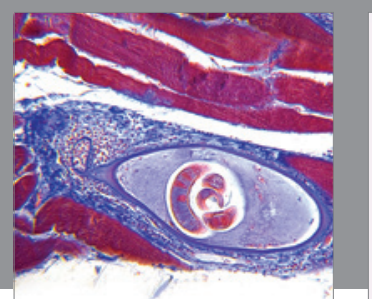

Gastroenterology Research and Practice

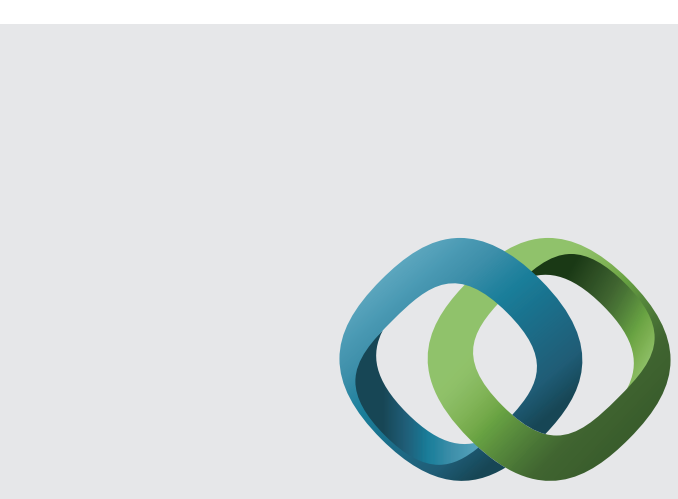

\section{Hindawi}

Submit your manuscripts at

http://www.hindawi.com
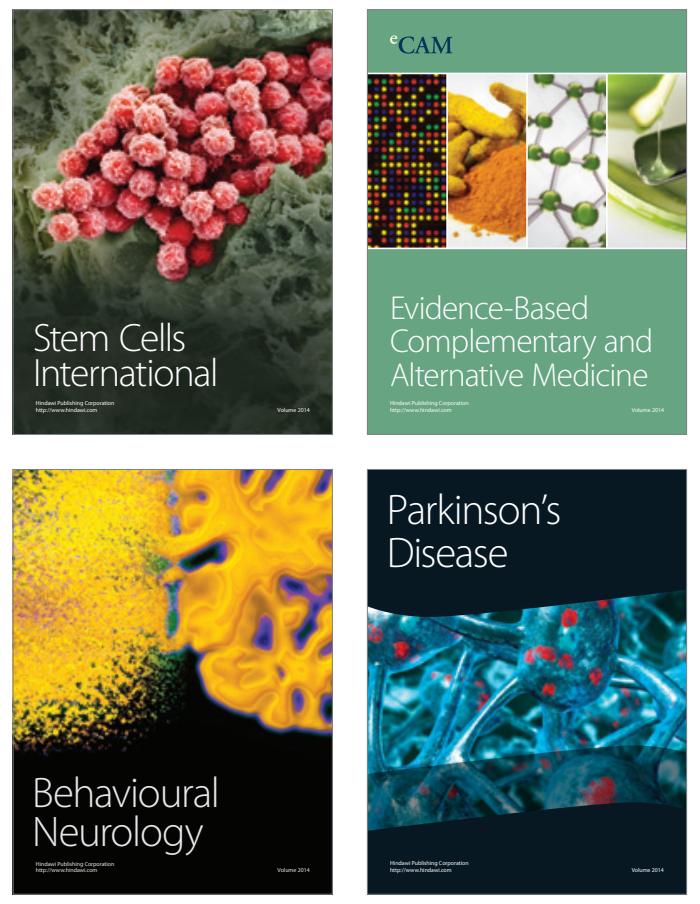
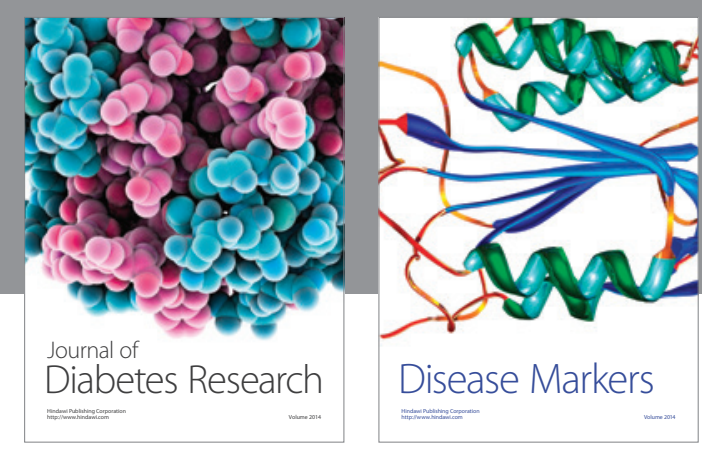

Disease Markers
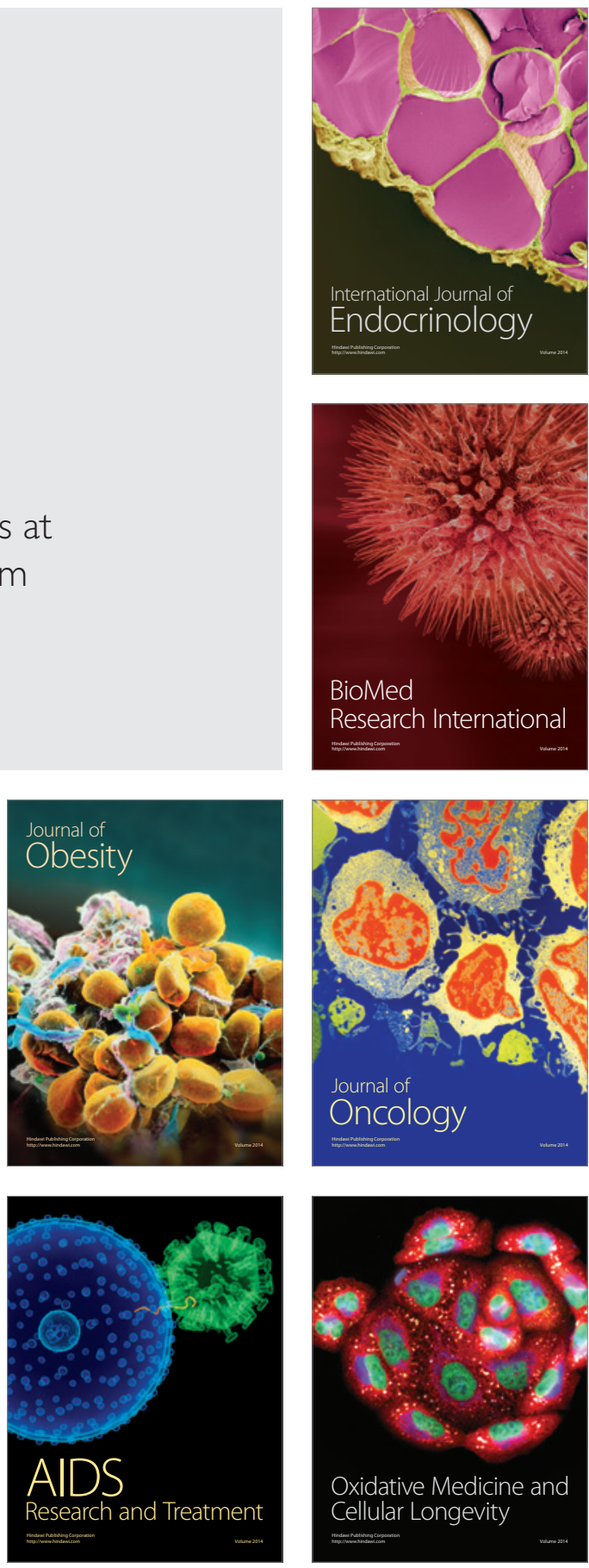